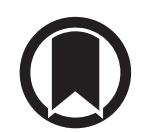

CrossMark

\title{
Outcome of liver transplantation for hepatopulmonary syndrome: a Eurotransplant experience
}

\author{
To the Editor:
}

Hepatopulmonary syndrome (HPS) is a pulmonary vascular complication of liver disease that affects up to $30 \%$ of patients with cirrhosis [1]. Intrapulmonary vascular dilatations and shunts result in gas exchange abnormalities, ranging from elevated alveolar-arterial oxygen gradients with no hypoxaemia to very severe hypoxaemia [1, 2]. Currently, liver transplantation (LT) is the only treatment option [3]. The Model for End-Stage Liver Disease (MELD) is a scoring system for assessing liver disease severity that has been validated to predict the 3-month waiting list mortality and is used by Eurotransplant for prioritising allocation of liver transplants [4]. However, this score poorly predicts overall and post-transplant survival, and does not take into account complications that affect outcomes independent of liver disease severity [5]. Hypoxaemia in HPS is generally progressive and mortality is highest in advanced stages [6, 7]. In this sense, a standard exception (SE) policy has been established to prioritise patients with severe HPS (arterial oxygen tension $\left(\mathrm{PaO}_{2}\right)<60 \mathrm{mmHg}$ ), as their severity of illness is not properly reflected by the MELD score. In the pre-SE MELD era, FALLON et al. [1] reported that HPS is associated with a doubled risk of mortality compared to patients without HPS. In 2014, GOLDBERG et al. [5] reviewed SE LT outcomes in HPS patients in the USA and found that LT candidates with SE for HPS had decreased pre-transplantation mortality and superior overall survival compared to non-HPS patients. The European outcomes for patients with SE for HPS have never been explored. In this retrospective study, we analysed overall, pre-transplant and post-transplant survival in LT candidates with SE for HPS within Eurotransplant, and determined whether the intent of the exception policy is being met.

All analyses used anonymised data available through the Eurotransplant registry from January 1, 2006, until December 31, 2013, comprising patients from Germany, Belgium, Austria, the Netherlands, Croatia, Hungary and Slovenia. The HPS cohort included all waiting list candidates aged $\geqslant 18$ years registered for their first LT with SE approved by Eurotransplant, according to disease- and country-specific criteria [8]. The exceptional MELD is expressed as percentage 3-month probability of death on the waiting list. Patients with approved SE for HPS are granted an initial SE MELD compatible with a 3-month probability of death of 15\% (a score of 22) in Austria, Belgium, Luxemburg, Germany, Slovenia and Croatia, and 10\% (a score of 20) in the Netherlands. This exceptional MELD is reconfirmed every 90 days and an update of $+10 \%$ MELD equivalent applies in all Eurotransplant countries. The non-HPS group consisted of waitlist candidates without any exception who were matched to the HPS cases (propensity score matching, 5:1 ratio) based on age, sex, aetiology of liver disease and MELD score at the time of listing. Statistical analyses were performed using SPSS 25 (SPSS, Inc., Chicago, IL, USA) and R3.4.1 software packages (R Foundation for Statistical Computing, Vienna, Austria). The Eurotransplant Liver and Intestine Advisory Committee and the ethical committee of the Faculty of Medicine and Health Sciences, Ghent University (Ghent, Belgium) approved the study protocol (2014/0927).

The study population consisted of 88 patients with SE for severe HPS and 442 non-HPS patients. Cox regression showed that overall mortality was not statistically different in HPS (hazard ratio (HR) 1.32, 95\% CI 0.93-1.88; $\mathrm{p}=0.13$ ) versus non-HPS patients (figure 1a). Fine and Gray regression models were used to evaluate pre-transplantation outcome, considering transplantation as a competing risk [9]. Pre-transplant mortality risk was similar in HPS and non-HPS waiting list candidates (HR 0.88, 95\% CI 0.52-1.47; $\mathrm{p}=0.62$ ) (figure $1 \mathrm{~b})$. A total of $128(24 \%)$ patients died on the waiting list: 17 (19\%) out of 88 HPS and

@ERSpublications

Equal overall survival among liver transplantation candidates supports current prioritisation policy for severe hepatopulmonary syndrome http://ow.ly/4SQS30mwOtK

Cite this article as: Raevens S, Rogiers X, Geerts A, et al. Outcome of liver transplantation for hepatopulmonary syndrome: a Eurotransplant experience. Eur Respir J 2019; 53: 1801096 [https://doi.org/ 10.1183/13993003.01096-2018]. 

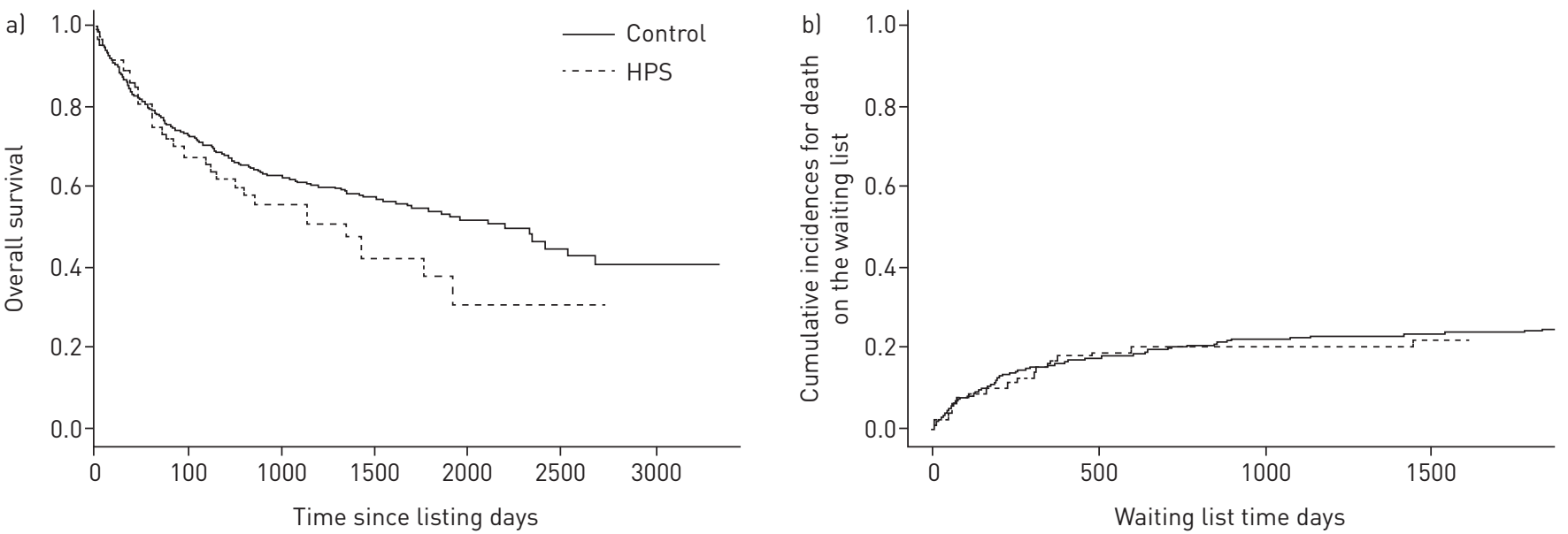

FIGURE 1 a) Overall patient survival of hepatopulmonary syndrome (HPS) versus non-HPS waiting list candidates. b) Competing risk curves for pre-transplantation waitlist survival in HPS versus non-HPS waiting list candidates. Unadjusted subdistribution hazard ratio for HPS 0.88 (95\% $\mathrm{Cl}$ $0.52-1.47, p=0.62)$

$111(25 \%)$ out of 442 non-HPS patients. Causes of death did not differ between groups and mainly included infections (11 out of 17 HPS and 58 out of 111 non-HPS) and progression of liver disease (two out of 17 HPS and 13 out of 111 non-HPS). Patients with HPS were prioritised for transplantation relative to patients without HPS due to the SE policy (HR 1.37, 95\% CI 1.04-1.80; $\mathrm{p}=0.026$ ). $69 \%$ of HPS patients received a transplant versus $54 \%$ of the non-HPS patients in the study period. $3 \%$ of patients in both groups were removed from the waiting list because they were too sick to be transplanted (three out of 88 HPS and 14 out of 442 non-HPS), and one (1\%) HPS patient and 45 (10\%) non-HPS patients were removed because their clinical status had improved.

Overall, 80 (27\%) out of 298 transplanted patients had died at the time of data analysis (24 (39\%) out of 61 HPS versus $56(24 \%)$ out of 237 non-HPS; $\mathrm{p}=0.014)$. The median post-LT follow-up for HPS patients was 2 years. Survival analysis demonstrated 1- and 3-month post-LT survival rates of respectively $91 \%$ (95\% CI $83-99 \%)$ and $84 \%$ (95\% CI 74-96\%) in HPS versus $96 \%$ (95\% CI 93-98\%) and 89\% (95\% CI $85-94 \%)$ in non-HPS patients. Death in the early post-operative period was primarily caused by infections (57\% HPS and 50\% non-HPS deaths). One HPS patient died because of respiratory insufficiency. Post-LT survival rates were 77\% (95\% CI 66-91\%) in HPS and 85\% (95\% CI 81-90\%) in non-HPS at 6 months, $70 \%(95 \%$ CI $57-85 \%)$ in HPS and $81 \%(95 \%$ CI $75-86 \%)$ in non-HPS at 1 year, and 64\% (95\% CI $51-80 \%)$ in HPS and $77 \%$ (95\% CI $71-83 \%)$ in non-HPS patients at 2 years after LT. Drop-out at later time-points post-transplant was more frequent in the HPS group (17 out of 61 versus 36 out of 237 in non-HPS), although causes of death did not differ between groups (mainly infections; $\mathrm{p}=0.275$ ).

We present the first international analysis of the outcome of LT candidates with SE for HPS in Europe. Two observations have direct clinical importance. First, although cases with HPS had a greater chance of receiving a transplant, overall mortality, which is the most important measure of equity between patient groups, did not differ between LT candidates with HPS and those without. These data indicate that since the implementation of a SE policy for HPS, the outcome has improved in this specific patient population compared to the pre-SE era [1], which concurs with the conclusion from the most recent and largest analysis in the USA [5]. However, in contrast, observations in the USA even indicated an overall survival benefit for HPS compared to non-HPS patients [5]. This was due to decreased pre-transplantation mortality in patients with HPS and suggested that the current exception policy may overprioritise HPS patients. Pre-transplant mortality risk was equal in both groups in our study, which, combined with similar overall survival, advocates against modification of current HPS exception policy. Defining a lower limit of $\mathrm{PaO}_{2}$ for granting SE would result in increased waiting time, during which HPS may aggravate, and which ultimately may result in worse overall outcome.

Second, statistical analysis demonstrated that post-transplantation survival in patients with HPS is acceptable but less favourable relative to patients without HPS. These data should, however, be interpreted with caution. Median follow-up time in the HPS cohort was rather short (2 years), resulting in a significant amount of censored cases beyond this time-point, and as such limits drawing conclusions with regard to long-term post-transplant survival. Nonetheless, up to 2 years post-transplant, survival was comparable in both groups, and in agreement with results from previous studies [7, 10-12]. Moreover, even beyond this period, causes of death did not differ. 
Our observations are different from those reported in the United Network for Organ Sharing (UNOS) zone [5], although decision-making with regard to transplant and the SE policy for HPS are similar in Eurotransplant [8] and UNOS [13]. In general, survival rates are lower in the Eurotransplant region compared to UNOS, which has been recognised before, and is explained by lower donor quality. The mean donor risk index (DRI), a metric of donor quality, is significantly higher in Eurotransplant, where $>50 \%$ of organs are considered "suboptimal", versus UNOS $[14,15]$. Only $<6 \%$ of donor livers in the USA were reported to have a DRI $>2$, where organs of marginal quality are more frequently discarded, versus $23 \%$ in Eurotransplant [15].

Lastly, although SE criteria for HPS within Eurotransplant are limited to cases with severe HPS, and as such only patients with $\mathrm{PaO}_{2}<60 \mathrm{mmHg}$ were included in this study, the exact values were not available through the registry. Consequently, the relationship between pre-LT oxygenation and post-LT survival could not be evaluated in this HPS cohort, which we acknowledge as a limitation to this study.

In conclusion, our results indicate that waitlist mortality and post-transplant survival in patients with severe HPS are fairly balanced under current SE policy, without disadvantaging the general transplant population.

Sarah Raevens ${ }^{1}$, Xavier Rogiers ${ }^{2,3}$, Anja Geerts ${ }^{1}$, Xavier Verhelst ${ }^{1}$, Undine Samuel ${ }^{3}$, Marieke van Rosmalen ${ }^{3}$, Gabriela Berlakovich ${ }^{4}$, Jean Delwaide ${ }^{5}$, Olivier Detry $\oplus^{6}$, Frank Lehner ${ }^{7}$, Jens Mittler ${ }^{8}$, Silvio Nadalin ${ }^{9}$, Frederik Nevens $\oplus^{10}$, Jacques Pirenne ${ }^{11}$, Fuat Saner ${ }^{12}$, Stefan Schneeberger ${ }^{13}$, Dirk Stippel ${ }^{14}$, Jerovšek Marjana Turk ${ }^{15}$, Mathe Zoltan ${ }^{16}$, Roberto Ivan Troisi ${ }^{2,17}$, Hans Van Vlierberghe ${ }^{1}$ and Isabelle Colle ${ }^{1}$

${ }^{1}$ Dept of Gastroenterology and Hepatology, Ghent University Hospital, Ghent University, Ghent, Belgium. ${ }^{2}$ Dept of General, Hepatobiliary and Liver Transplantation Surgery, Ghent University Hospital, Ghent University, Ghent, Belgium. ${ }^{3}$ Eurotransplant, Leiden, The Netherlands. ${ }^{4}$ Division of Transplantation, Dept of Surgery, Medical University of Vienna, Vienna, Austria. ${ }^{5}$ Dept of Gastroenterology and Hepatology, CHU Liège, University of Liège, Liège, Belgium. ${ }^{6}$ Dept of Abdominal Surgery and Transplantation, CHU Liège, University of Liège, Liège, Belgium. ${ }^{7}$ General, Visceral and Transplant Surgery, Hannover Medical School, Hannover, Germany. ${ }^{8}$ Dept of General, Visceral, and Transplant Surgery, University Medical Center Mainz, Mainz, Germany. ${ }^{9}$ Dept of General, Visceral and Transplant Surgery, University Hospital Tübingen, Tübingen, Germany. ${ }^{10}$ Dept of Gastroenterology and Hepatology, University Hospitals KU Leuven, Leuven, Belgium. ${ }^{11}$ Abdominal Transplant Surgery, University Hospitals KU Leuven, Leuven, Belgium. ${ }^{12}$ Dept of General, Visceral and Transplant Surgery, University Hospital Essen, Essen, Germany. ${ }^{13}$ Dept of Visceral Transplant and Thoracic Surgery, Medical University Innsbruck, Innsbruck, Austria. ${ }^{14}$ Division of Transplantation Surgery, Dept of General, Visceral and Cancer Surgery, Transplant Center Cologne, University of Cologne, Cologne, Germany. ${ }^{15}$ Dept of Gastroenterology and Hepatology, University Medical Center Ljubljana, Ljubljana, Slovenia. ${ }^{16}$ Dept of Transplantation and Surgery, Semmelweis University Budapest, Budapest, Hungary. ${ }^{17}$ Dept of Clinical Medicine and Surgery, Federico II University Naples, Naples, Italy.

Correspondence: Sarah Raevens, Dept of Gastroenterology and Hepatology, Ghent University Hospital, Building K12, First Floor IE, Corneel Heymanslaan 10, 9000 Ghent, Belgium. E-mail: sarah.raevens@ugent.be

Received: June 122018 | Accepted after revision: Oct 222018

Acknowledgements: The authors thank Roos Colman (Dept of Public Health, Biostatistics Unit, Ghent University, Ghent, Belgium) for her assistance in the statistical analysis of the data, the Eurotransplant representatives for supporting the organisation of this work and all Eurotransplant liver transplantation centres for providing data to the Eurotransplant registry.

Conflict of interest: S. Raevens has nothing to disclose. X. Rogiers has nothing to disclose. A. Geerts has nothing to disclose. X. Verhelst has nothing to disclose. U. Samuel has nothing to disclose. M. van Rosmalen has nothing to disclose. G. Berlakovich has nothing to disclose. J. Delwaide has nothing to disclose. O. Detry has nothing to disclose. F. Lehner has nothing to disclose. J. Mittler has nothing to disclose. S. Nadalin has nothing to disclose. F. Nevens reports receiving grants from Roche, Astellas and Sandoz, grants and consultancy fees from BMS, CAF, MSD, TwinPharma and Ipsen, and consultancy fees from Gilead, Novartis, Abbvie, Promethera Biosciences, Durect, Ferring, Gore, Cook Medical, Biotest and Intercept, outside the submitted work. J. Pirenne has nothing to disclose. F. Saner has nothing to disclose. S. Schneeberger reports being a member of an expert group and a consultant for Merck; being a member of an expert group and a speaker for Astellas; receiving grants, and being a member of an expert group and a consultant for Chiesi; being a member of an expert group, a speaker and a consultant for Teva; being a speaker for Novartis; and receiving grants from Sandoz, all outside the submitted work. D. Stippel has nothing to disclose. M. Turk Jerovšek has nothing to disclose. M. Zoltan has nothing to disclose. R.I. Troisi has nothing to disclose. H. Van Vlierberghe has nothing to disclose. I. Colle reports consultancy for Promethera outside the submitted work.

Support statement: S. Raevens is paid by a fellowship from the Research Foundation - Flanders (11W5715N). Funding information for this article has been deposited with the Crossref Funder Registry.

\section{References}

1 Fallon MB, Krowka MJ, Brown RS, et al. Impact of hepatopulmonary syndrome on quality of life and survival in liver transplant candidates. Gastroenterology 2008; 135: 1168-1175.

2 Raevens S, Geerts A, Van Steenkiste C, et al. Hepatopulmonary syndrome and portopulmonary hypertension: recent knowledge in pathogenesis and overview of clinical assessment. Liver Int 2015; 35: 1646-1660. 
3 Krowka MJ, Porayko MK, Plevak DJ, et al. Hepatopulmonary syndrome with progressive hypoxemia as an indication for liver transplantation: case reports and literature review. Mayo Clin Proc 1997; 72: 44-53.

4 Wiesner R, Edwards E, Freeman R, et al. Model for end-stage liver disease (MELD) and allocation of donor livers. Gastroenterology 2003; 124: 91-96.

5 Goldberg DS, Krok K, Batra S, et al. Impact of the hepatopulmonary syndrome MELD exception policy on outcomes of patients after liver transplantation: an analysis of the UNOS database. Gastroenterology 2014; 146: $1256-1265$.

6 Arguedas MR, Abrams GA, Krowka MJ, et al. Prospective evaluation of outcomes and predictors of mortality in patients with hepatopulmonary syndrome undergoing liver transplantation. Hepatology 2003; 37: 192-197.

7 Swanson KL, Wiesner RH, Krowka MJ. Natural history of hepatopulmonary syndrome: impact of liver transplantation. Hepatology 2005; 41: 1122-1129.

8 Eurotransplant. Eurotransplant manual. Leiden, Eurotransplant International Foundation, 2016.

9 Kim WR, Therneau TM, Benson JT, et al. Deaths on the liver transplant waiting list: an analysis of competing risks. Hepatology 2006; 43: 345-351.

10 Taille C, Cadranel J, Bellocq A, et al. Liver transplantation for hepatopulmonary syndrome: a ten-year experience in Paris, France. Transplantation 2003; 75: 1482-1489.

11 Kim HY, Choi MS, Lee SC, et al. Outcomes in patients with hepatopulmonary syndrome undergoing liver transplantation. Transplant Proc 2004; 36: 2762-2763.

12 Iyer VN, Swanson KL, Cartin-Ceba R, et al. Hepatopulmonary syndrome: favorable outcomes in the MELD exception era. Hepatology 2013; 57: 2427-2435.

13 Organ Procurement and Transplantation Network. Policy 9: Allocation of livers and liver-intestines. https://optn. transplant.hrsa.gov/media/1200/optn_policies.pdf. Date last updated: September 1, 2018. Date last accessed: September 6, 2018

14 Blok JJ, Braat AE, Adam R, et al. Validation of the donor risk index in orthotopic liver transplantation within the Eurotransplant region. Liver Transpl 2012; 18: 112-119.

15 Schlitt HJ, Loss M, Scherer MN, et al. Aktuelle Entwicklungen der Lebertransplantation in Deutschland: MELD-basierte Organallokation und "incentives" für Transplantationszentren [Current developments in liver transplantation in Germany: MELD-based organ allocation and incentives for transplant centres]. Z Gastroenterol 2011; 49: 30-38. 